\title{
Robust Adaptive Output Feedback Control Scheme for Chaos Synchronization with Input Nonlinearity
}

\author{
Xiaomeng Li, ${ }^{1}$ Zhanshan Zhao, ${ }^{1}$ Jing Zhang, ${ }^{2,3}$ and Meixia Zhu ${ }^{1}$ \\ ${ }^{1}$ School of Computer Science \& Software Engineering, Tianjin Polytechnic University, Tianjin 300387, China \\ ${ }^{2}$ School of Textiles, Tianjin Polytechnic University, Tianjin 300387, China \\ ${ }^{3}$ Tianjin Vocational Institute, Tianjin 300410, China
}

Correspondence should be addressed to Zhanshan Zhao; zhzhsh127@163.com

Received 22 July 2015; Accepted 10 January 2016

Academic Editor: Hung-Yuan Chung

Copyright (C) 2016 Xiaomeng Li et al. This is an open access article distributed under the Creative Commons Attribution License, which permits unrestricted use, distribution, and reproduction in any medium, provided the original work is properly cited.

\begin{abstract}
This paper proposes a robust adaptive output feedback control strategy which can automatically regulate control gain for chaos synchronization. Chaotic systems with input nonlinearities, delayed nonlinear coupling, and external disturbance can achieve synchronization by applying this strategy. Utilizing Lyapunov method and LMI technique, the conditions ensuring chaos synchronization are obtained. Finally, simulations are given to show the effectiveness of our control strategy.
\end{abstract}

\section{Introduction}

Chaos synchronization has attracted a lot of interest due to its wide engineering application in various areas like secure communication [1-4], neural networks [5, 6], electronic engineering [7], and so on $[8,9]$. Consider the fact that chaotic system is a class of nonlinear dynamical system which sensitively depends on initial conditions. It is necessary to solve the problem of chaos synchronization.

In the practical physical systems, physical limitations will lead to state nonlinearity and input nonlinearity, such as sector [10-12], saturation [13-15], and dead zones [16-19]. Considering state nonlinearity, [20] proposed an adaptive control strategy for multirate networked nonlinear systems. In [21], fuzzy control method has been applied to a class of nonlinear system. Filter design and $H_{\infty}$ performance for nonlinear networked systems have been researched in [22]. A novel sliding mode observer approach has been proposed for a class of stochastic systems in [23]. Moreover, it should not be ignored that effect of nonlinear control inputs can cause serious degradation of synchronization performance even nonsynchronous. Thus, nonlinear control inputs should be considered in synchronization controller design of chaotic systems. Unfortunately, in $[24,25]$, input nonlinearity has not been considered.
As a source of nonsynchronism, time-varying delay has to be faced in many engineering synchronization systems, such as chemical processes $[26,27]$ and pneumatic systems [28]. Therefore, designing a controller for time-varying delay systems is necessary [29].

Recently, considering nonlinearly coupled chaotic systems, [30] proposed a state feedback controller to achieve synchronization. However, the input nonlinearity was not considered. To the authors' knowledge, synchronization for coupled chaotic systems with input nonlinearity and time delays has been rarely mentioned. Furthermore, in real application, only the output state is available. Therefore, it is necessary to design a synchronization controller in output feedback form.

Motivated by the previous discussions, we propose an adaptive output feedback controller to make chaotic systems synchronize. Input nonlinearities, nonlinear coupling, and time-varying delay have been taken into account. By utilizing Lyapunov method and LMI technique, the conditions ensuring synchronization are obtained.

In the rest of this paper, Section 2 provides systems and problem description. Then a robust adaptive output feedback control strategy is proposed for chaos synchronization in Section 3. In Section 4, simulations are given to demonstrate 
effectiveness of this control strategy. Conclusion are collected in Section 5.

\section{Problem Formulation}

Consider chaotic systems as follows:

$$
\begin{aligned}
\dot{x}_{m}(t)= & A x_{m}(t)+A_{c} x_{m}\left(t-d_{1}(t)\right)+B f\left(t, x_{m}(t)\right) \\
& +B_{c} g\left(t, x_{m}\left(t-d_{1}(t)\right)\right) \\
& -B_{d} k\left(t, x_{s}\left(t-d_{2}(t)\right)\right), \\
y_{m}(t)= & C x_{m}(t) \\
\dot{x}_{s}(t)= & A x_{s}(t)+A_{c} x_{s}\left(t-d_{1}(t)\right)+B f\left(t, x_{s}(t)\right) \\
& +B_{c} g\left(t, x_{s}\left(t-d_{1}(t)\right)\right) \\
& -B_{d} k\left(t, x_{m}\left(t-d_{2}(t)\right)\right)+D \omega(t) \\
& +E \Lambda(u(t)), \\
y_{s}(t)= & C x_{s}(t),
\end{aligned}
$$

where $x_{m} \in \mathbb{R}^{n}, x_{s} \in \mathbb{R}^{n}, y_{m} \in \mathbb{R}^{m}$, and $y_{s} \in \mathbb{R}^{m}$ are the state vector and output vector for the drive and response system, respectively. $f(t, x), g(t, x), k(t, x): \mathbb{R}^{n} \times \mathbb{R}^{n} \rightarrow \mathbb{R}^{m}$ represent nonlinear vectors. $u(t)=\left[u_{1}(t) \cdots u_{m}(t)\right]^{T} \in \mathbb{R}^{m}$ is the control input vector; $\omega(t)$ denotes the external disturbance; time-varying delay $d_{1}(t)$ and $d_{2}(t)$ satisfy

$$
\begin{aligned}
& d_{11} \leq \dot{d}_{1}(t) \leq d_{12} \\
& d_{21} \leq \dot{d}_{2}(t) \leq d_{22} .
\end{aligned}
$$

$\Lambda(u(t))=\left[\lambda_{1}\left(u_{1}(t)\right) \cdots \lambda_{m}\left(u_{m}(t)\right)\right]^{T}$ is representing the nonlinear control input vector which satisfies the following inequality:

$$
\nu_{i}(t) \lambda_{i}\left(\nu_{i}(t)\right) \geq \chi_{i}\left(\nu_{i}(t)\right)^{2}
$$

$\chi_{i}$ is an unknown positive constant satisfying $\chi^{*}=\min \chi_{i}$. Constant matrices $A, A_{c}, B, B_{c}, B_{d}, C, D, E$ have appropriate dimensions.

Synchronization error can be defined as $e(t)=x_{s}(t)-$ $x_{m}(t)$. Using (1) and (2), synchronization error can be obtained:

$$
\begin{aligned}
\dot{e}(t)= & A e(t)+A_{c} e\left(t-d_{1}(t)\right)+B \Psi(t)+B_{c} \Upsilon\left(t, d_{1}\right) \\
& +B_{d} \Xi\left(t, d_{2}\right)+D \omega(t)+E \Lambda(u(t)), \\
y_{e}(t)= & C e(t),
\end{aligned}
$$

where

$$
\begin{aligned}
\Psi(t) & =f\left(t, x_{s}(t)\right)-f\left(t, x_{m}(t)\right), \\
\Upsilon\left(t, d_{1}\right) & =g\left(t, x_{s}\left(t-d_{1}(t)\right)\right)-g\left(t, x_{m}\left(t-d_{1}(t)\right)\right), \\
\Xi\left(t, d_{2}\right) & =k\left(t, x_{s}\left(t-d_{2}(t)\right)\right)-k\left(t, x_{m}\left(t-d_{2}(t)\right)\right) .
\end{aligned}
$$

The objective is to make drive and response systems synchronize. Obviously, if $e(t) \rightarrow 0$, then $x_{s}(t)-x_{m}(t) \rightarrow 0$ and it means that system (1) and (2) is synchronized.

To obtain the synchronization conditions, the following lemma and assumptions will be used during the proof.

Lemma 1. If matrix $H=\left[\begin{array}{cc}H_{11} & H_{12} \\ H_{21} & H_{22}\end{array}\right]$, where $H_{11}$ and $H_{22}$ are square matrices, then the following inequalities are equivalent:

(1) $H<0$;

(2) $H_{11}<0, H_{22}-H_{12}^{T} H_{11}^{-1} H_{12}<0$;

(3) $H_{22}<0, H_{11}-H_{12} H_{22}^{-1} H_{12}^{T}<0$.

Assumption 2. The nonlinear function $\Psi(t), \Upsilon\left(t, d_{1}\right)$, and $\Xi\left(t, d_{2}\right)$ satisfy the global Lipschitz condition:

$$
\begin{aligned}
\|\Psi(t)\| & \leq L_{1}\left\|x_{s}(t)-x_{m}(t)\right\|, \\
\left\|\Upsilon\left(t, d_{1}\right)\right\| & \leq L_{2}\left\|x_{s}\left(t-d_{1}(t)\right)-x_{m}\left(t-d_{1}(t)\right)\right\|, \\
\left\|\Xi\left(t, d_{2}\right)\right\| & \leq L_{3}\left\|x_{s}\left(t-d_{2}(t)\right)-x_{m}\left(t-d_{2}(t)\right)\right\| .
\end{aligned}
$$

Assumption 3. Matrix $P>0$ and satisfies the following equation:

$$
E^{T} P=C
$$

\section{Robust Adaptive Controller Design Based on LMI}

In order to make drive and response systems synchronize, the following adaptive controller is considered:

$$
u(t)=-\frac{v}{2} C e(t)
$$

where $v$ is an adaptive control gain and is adjusted by the following adaptation law:

$$
\dot{v}=\chi^{*} \rho\|C e(t)\|^{2}, \quad \nu(0)>0,
$$

where $\rho$ is a positive parameter.

By applying of the adaptive controller, synchronization errors will converge to zero asymptotically. In Theorems 4 and 5 the main results will be presented. 
Theorem 4. Consider the drive and response system (1) and (2) under $\omega(t)=0$. By application of the adaptive control law (10) and (11), if existing symmetric and positive definite matrices $P$, $W_{1}, W_{2}$, and a scalar $\alpha>0$, satisfying the following $L M I$ :

$$
\left[\begin{array}{ccccccccc}
\Delta & P A_{c} & 0 & P B & P B_{c} & P B_{d} & L_{1}^{T} & 0 & 0 \\
* & -\left(1-d_{12}\right) W_{1} & 0 & 0 & 0 & 0 & 0 & L_{2}^{T} & 0 \\
* & * & -\left(1-d_{22}\right) W_{2} & 0 & 0 & 0 & 0 & 0 & L_{3}^{T} \\
* & * & * & -I & 0 & 0 & 0 & 0 & 0 \\
* & * & * & * & -I & 0 & 0 & 0 & 0 \\
* & * & * & * & * & -I & 0 & 0 & 0 \\
* & * & * & * & * & * & -I & 0 & 0 \\
* & * & * & * & * & * & * & -I & 0 \\
* & * & * & * & * & * & * & * & -I
\end{array}\right]<0,
$$

where

$$
\Delta=P A+A^{T} P-\alpha P E E^{T} P+W_{1}+W_{2},
$$

the system (1) and (2) is synchronized.

Proof. We consider the following Lyapunov-Krasovskii functional:

$$
\begin{aligned}
V= & e^{T}(t) P e(t)+\int_{t-d_{1}(t)}^{t} e^{T}(v) W_{1} e(v) d v \\
& +\int_{t-d_{2}(t)}^{t} e^{T}(v) W_{2} e(v) d v+\frac{1}{2} \rho^{-1} \widetilde{v}^{2},
\end{aligned}
$$

where $\widetilde{\nu}=v^{*}-v \cdot v^{*}$ and $\rho$ are positive constants.

The derivative of $V$ can be calculated as follows:

$$
\begin{aligned}
\dot{V} & =2 e^{T}(t) P \dot{e}(t)+e^{T}(t) W_{1} e(t)-\left(1-\dot{d}_{1}(t)\right) e^{T}(t \\
& \left.-d_{1}(t)\right) W_{1} e\left(t-d_{1}(t)\right)+e^{T}(t) W_{2} e(t)-(1 \\
& \left.-\dot{d}_{2}(t)\right) e^{T}\left(t-d_{2}(t)\right) W_{2} e\left(t-d_{2}(t)\right)-\rho^{-1} \dot{\nu} \widetilde{\nu} \\
& =2 e^{T}(t) P\left[A e(t)+A_{c} e\left(t-d_{1}(t)\right)+B \Psi(t)\right. \\
& \left.+B_{c} \Upsilon\left(t, d_{1}\right)+B_{d} \Xi\left(t, d_{2}\right)+E \Lambda(u(t))\right]+e^{T}(t) \\
& -W_{1} e(t)-\left(1-\dot{d}_{1}(t)\right) e^{T}\left(t-d_{1}(t)\right) W_{1} e(t \\
& \left.-d_{1}(t)\right)+e^{T}(t) W_{2} e(t)-\left(1-\dot{d}_{2}(t)\right) e^{T}(t \\
& \left.-d_{2}(t)\right) W_{2} e\left(t-d_{2}(t)\right)-\rho^{-1} \dot{\nu} \widetilde{v} .
\end{aligned}
$$

Incorporating (3) and (4), we can get

$$
\begin{aligned}
\dot{V} \leq & e^{T}(t)\left(P A+A^{T} P-\alpha P E E^{T} P+W_{1}+W_{2}\right) e(t) \\
& +\alpha\left\|E^{T} P e(t)\right\|^{2}+2 e^{T}(t) P A_{c} e\left(t-d_{1}(t)\right) \\
& +2 e^{T}(t) P B \Psi(t)+2 e^{T}(t) P B_{c} \Upsilon\left(t, d_{1}\right) \\
& +2 e^{T}(t) P B_{d} \Xi\left(t, d_{2}\right)+2 e^{T}(t) P E \Lambda(u(t)) \\
& -\left(1-d_{12}\right) e^{T}\left(t-d_{1}(t)\right) W_{1} e\left(t-d_{1}(t)\right) \\
& -\left(1-d_{22}\right) e^{T}\left(t-d_{2}(t)\right) W_{2} e\left(t-d_{2}(t)\right) \\
& -\rho^{-1} \dot{v} \widetilde{v} .
\end{aligned}
$$

Using (8) leads to

$$
\begin{aligned}
\dot{V} \leq & e^{T}(t)\left(P A+A^{T} P-\alpha P E E^{T} P+W_{1}+W_{2}\right) e(t) \\
& +\alpha\left\|E^{T} P e(t)\right\|^{2}+2 e^{T}(t) P A_{c} e\left(t-d_{1}(t)\right) \\
& +2 e^{T}(t) P B \Psi(t)+2 e^{T}(t) P B_{c} \Upsilon\left(t, d_{1}\right) \\
& +2 e^{T}(t) P B_{d} \Xi\left(t, d_{2}\right)+2 e^{T}(t) P E \Lambda(u(t)) \\
& -\left(1-d_{12}\right) e^{T}\left(t-d_{1}(t)\right) W_{1} e\left(t-d_{1}(t)\right) \\
& -\left(1-d_{22}\right) e^{T}\left(t-d_{2}(t)\right) W_{2} e\left(t-d_{2}(t)\right) \\
& -\rho^{-1} \dot{\nu} \widetilde{\nu}+e^{T}(t) L_{1}^{T} L_{1} e(t) \\
& +e^{T}\left(t-d_{1}(t)\right) L_{2}^{T} L_{2} e\left(t-d_{1}(t)\right) \\
& +e^{T}\left(t-d_{2}(t)\right) L_{3}^{T} L_{3} e\left(t-d_{2}(t)\right)-\Psi^{T}(t) \Psi(t) \\
& -\Upsilon^{T}\left(t, d_{1}\right) \Upsilon\left(t, d_{1}\right)-\Xi^{T}\left(t, d_{2}\right) \Xi\left(t, d_{2}\right) .
\end{aligned}
$$


Assume $\mathrm{Ce}(t)=Z(t)_{m \times 1} ; Z_{n}(t)$ is the $n$th element of $Z(t)$. By using (11), it is easy to prove that $\nu>0$. Based on (5), we need to consider the following two cases:

(1) When $Z_{n}(t)>0$, we have $u_{n}(t)<0$. Multiplying $Z_{n}(t)>0$ and dividing $u_{n}(t)<0$ by both sides of (5), we can obtain that $Z_{n}(t) \lambda_{n}\left(\nu_{n}(t)\right) \leq \chi_{n} Z_{n}(t) \nu_{n}(t)$.

(2) When $Z_{n}(t)<0$, we have $u_{n}(t)>0$. Multiplying $Z_{n}(t)<0$ and dividing $u_{n}(t)>0$ by both sides of (5), we can obtain that $Z_{n}(t) \lambda_{n}\left(\nu_{n}(t)\right) \leq \chi_{n} Z_{n}(t) \nu_{n}(t)$.

Form the previous discussion, we can prove that the following relation always holds:

$$
Z_{n}(t) \lambda_{n}\left(v_{n}(t)\right) \leq \chi_{n} Z_{n}(t) v_{n}(t)
$$

Using Assumption 3 and $\chi^{*}=\min \chi_{n}$ we have

$$
\begin{aligned}
2 e^{T}(t) P E \Lambda(\nu(t)) & =2 \sum_{n=1}^{m} Z_{n}(t) \lambda_{n}\left(\nu_{n}(t)\right) \\
& \leq 2 \sum_{n=1}^{m} \chi_{n} Z_{n}(t) \nu_{n}(t) \\
& \leq-\chi^{*} v\|C e(t)\|^{2} .
\end{aligned}
$$

Let $\alpha=\chi^{*} \nu^{*}$; incorporating the previous result (19), we can obtain

$$
\begin{aligned}
\dot{V} & \leq e^{T}(t) \\
& \cdot\left(P A+A^{T} P-\alpha P E E^{T} P+W_{1}+W_{2}+L_{1}^{T} L_{1}\right) e(t)
\end{aligned}
$$

$$
\begin{aligned}
& +\chi^{*} \nu^{*}\|C e(t)\|^{2}+2 e^{T}(t) P A_{c} e\left(t-d_{1}(t)\right) \\
& +2 e^{T}(t) P B \Psi(t)+2 e^{T}(t) P B_{c} \Upsilon\left(t, d_{1}\right)+2 e^{T}(t) \\
& \cdot P B_{d} \Xi\left(t, d_{2}\right)-\chi^{*} \nu\|C e(t)\|^{2}-\left(1-d_{12}\right) \\
& \cdot e^{T}\left(t-d_{1}(t)\right) W_{1} e\left(t-d_{1}(t)\right)-\left(1-d_{22}\right) \\
& \cdot e^{T}\left(t-d_{2}(t)\right) W_{2} e\left(t-d_{2}(t)\right)-\chi^{*}\left(\nu^{*}-\nu\right) \\
& \cdot\|C e(t)\|^{2}+e^{T}\left(t-d_{1}(t)\right) L_{2}^{T} L_{2} e\left(t-d_{1}(t)\right) \\
& +e^{T}\left(t-d_{2}(t)\right) L_{3}^{T} L_{3} e\left(t-d_{2}(t)\right)-\Psi^{T}(t) \Psi(t) \\
& -\Upsilon^{T}\left(t, d_{1}\right) \Upsilon\left(t, d_{1}\right)-\Xi^{T}\left(t, d_{2}\right) \Xi\left(t, d_{2}\right)=e^{T}(t) \\
& \cdot\left(P A+A^{T} P-\alpha P E E^{T} P+W_{1}+W_{2}+L_{1}^{T} L_{1}\right) e(t) \\
& +e^{T}\left(t-d_{1}(t)\right)\left(L_{2}^{T} L_{2}-\left(1-d_{12}\right) W_{1}\right) \\
& \cdot e\left(t-d_{1}(t)\right)+e^{T}\left(t-d_{2}(t)\right) \\
& \cdot\left(L_{3}^{T} L_{3}-\left(1-d_{22}\right) W_{2}\right) e\left(t-d_{2}(t)\right)+2 e^{T}(t) \\
& \cdot P A_{c} e\left(t-d_{1}(t)\right)+2 e^{T}(t) P B \Psi(t)+2 e^{T}(t) \\
& \cdot P B_{c} \Upsilon\left(t, d_{1}\right)+2 e^{T}(t) P B_{d} \Xi\left(t, d_{2}\right)-\Psi^{T}(t) \Psi(t) \\
& \left.-d_{1}\right) \Upsilon\left(t, d_{1}\right)-\Xi^{T}\left(t, d_{2}\right) \Xi\left(t, d_{2}\right) \\
& \cdot(t)(t)
\end{aligned}
$$

which further can be written as $\dot{V} \leq \xi^{T} \Gamma_{1} \xi$, where

$$
\begin{aligned}
\xi^{T}= & {\left[\begin{array}{cccccc}
e^{T}(t) & e^{T}\left(t-d_{1}(t)\right) & e^{T}\left(t-d_{2}(t)\right) & \Psi^{T}(t) & \Upsilon^{T}\left(t, d_{1}\right) & \Xi^{T}\left(t, d_{2}\right)
\end{array}\right]^{T}, } \\
\Gamma_{1}= & {\left[\begin{array}{cccccc}
\Delta_{1} & P A_{c} & 0 & P B & P B_{c} & P B_{d} \\
* & \Delta_{2} & 0 & 0 & 0 & 0 \\
* & * & \Delta_{3} & 0 & 0 & 0 \\
* & * & * & -I & 0 & 0 \\
* & * & * & * & -I & 0 \\
* & * & * & * & * & -I
\end{array}\right]<0 }
\end{aligned}
$$

where

$$
\begin{aligned}
& \Delta_{1}=P A+A^{T} P-\alpha P E E^{T} P+L_{1}^{T} L_{1}+W_{1}+W_{2}, \\
& \Delta_{2}=L_{2}^{T} L_{2}-\left(1-d_{12}\right) W_{1}, \\
& \Delta_{3}=L_{3}^{T} L_{3}-\left(1-d_{22}\right) W_{2} .
\end{aligned}
$$

Using Lemma 1, (22) can be transformed to (12), which completed the proof of Theorem 4 .

In fact, disturbances always come from surrounding environment and communication channel. So, it is significant to solve the robustness problem in practical application. Theorem 5 will tackle this issue.

Theorem 5. Consider the drive and response system (1) and (2) under $\omega(t) \neq 0$. By application of the adaptive control law (10) and (11), for given $\gamma$, if existing symmetric and positive definite matrices $P, W_{1}, W_{2}$, and a scalar $\alpha>0$, satisfy the following LMI: 


$$
\left[\begin{array}{cccccccccc}
\Pi & P A_{c} & 0 & P B & P B_{c} & P B_{d} & P D & L_{1}^{T} & 0 & 0 \\
* & -\left(1-d_{12}\right) W_{1} & 0 & 0 & 0 & 0 & 0 & 0 & L_{2}^{T} & 0 \\
* & * & -\left(1-d_{22}\right) W_{2} & 0 & 0 & 0 & 0 & 0 & 0 & L_{3}^{T} \\
* & * & * & -I & 0 & 0 & 0 & 0 & 0 & 0 \\
* & * & * & * & -I & 0 & 0 & 0 & 0 & 0 \\
* & * & * & * & * & -I & 0 & 0 & 0 & 0 \\
* & * & * & * & * & * & -\gamma^{2} I & 0 & 0 & 0 \\
* & * & * & * & * & * & * & -I & 0 & 0 \\
* & * & * & * & * & * & * & * & -I & 0 \\
* & * & * & * & * & * & * & * & * & -I
\end{array}\right]<0,
$$

where

$$
\Pi=P A+A^{T} P-\alpha P E E^{T} P+W_{1}+W_{2}+C^{T} C,
$$

the attention rate $\gamma$ for $H_{\infty}$ synchronization in the disturbance situation can be achieved.

Proof. With zero initial condition, let us introduce

$$
J=\int_{o}^{\infty}\left[y_{e}^{T}(t) y_{e}(t)-\gamma^{2} \omega^{T}(t) \omega(t)\right] d t \leq 0
$$

For $\omega(t) \neq 0$, the following function can be obtained:

$$
J \leq \int_{o}^{\infty}\left[\dot{V}_{d}+y_{e}^{T}(t) y_{e}(t)-\gamma^{2} \omega^{T}(t) \omega(t)\right] d t \leq 0
$$

From the proof of Theorem $4, \dot{V}_{d}$ can be obtained:

$$
\begin{aligned}
\dot{V}_{d}=e^{T}(t) & \cdot\left(P A+A^{T} P-\alpha P E E^{T} P+L_{1}^{T} L_{1}+W_{1}+W_{2}\right) \\
\cdot & e(t)+e^{T}\left(t-d_{1}(t)\right)\left(L_{2}^{T} L_{2}-\left(1-d_{12}\right) W_{1}\right) \\
& \cdot e\left(t-d_{1}(t)\right)+e^{T}\left(t-d_{2}(t)\right) \\
& \cdot\left(L_{3}^{T} L_{3}-\left(1-d_{22}\right) W_{2}\right) e\left(t-d_{2}(t)\right)+2 e^{T}(t) \\
& \cdot P A_{c} e\left(t-d_{1}(t)\right)+2 e^{T}(t) P B \Psi(t)+2 e^{T}(t) \\
& \cdot P B_{c} \Upsilon\left(t, d_{1}\right)+2 e^{T}(t) P B_{d} \Xi\left(t, d_{2}\right)+2 e^{T}(t) \\
& \cdot P D \omega(t)-\Psi^{T}(t) \Psi(t)-\Upsilon^{T}\left(t, d_{1}\right) \Upsilon\left(t, d_{1}\right) \\
- & \Xi^{T}\left(t, d_{2}\right) \Xi\left(t, d_{2}\right) .
\end{aligned}
$$

Let

$$
\xi_{d}^{T}=\left[\begin{array}{lllllll}
e^{T}(t) & e^{T}\left(t-d_{1}(t)\right) & e^{T}\left(t-d_{2}(t)\right) & \omega^{T}(t) & \Psi^{T}(t) & \Upsilon^{T}\left(t, d_{1}\right) & \Xi^{T}\left(t, d_{2}\right)
\end{array}\right]^{T} .
$$

Equation (28) can be written as

$$
J \leq \xi_{d}^{T} \Gamma_{2} \xi_{d}^{T}
$$

where

$$
\Gamma_{2}=\left[\begin{array}{ccccccc}
\Pi_{1} & P A_{c} & 0 & P B & P B_{c} & P B_{d} & P D \\
* & \Pi_{2} & 0 & 0 & 0 & 0 & 0 \\
* & * & \Pi_{3} & 0 & 0 & 0 & 0 \\
* & * & * & -I & 0 & 0 & 0 \\
* & * & * & * & -I & 0 & 0 \\
* & * & * & * & * & -I & 0 \\
* & * & * & * & * & 0 & -\gamma^{2} I
\end{array}\right],
$$

(31)

$$
\begin{aligned}
\Pi_{1}= & P A+A^{T} P-\alpha P E E^{T} P+L_{1}^{T} L_{1}+W_{1}+W_{2} \\
& +C^{T} C
\end{aligned}
$$

$$
\begin{aligned}
& \Pi_{2}=L_{2}^{T} L_{2}-\left(1-d_{12}\right) W_{1}, \\
& \Pi_{3}=L_{3}^{T} L_{3}-\left(1-d_{22}\right) W_{2} .
\end{aligned}
$$

Using Lemma 1, (31) can be transformed to (24), which completed the proof of Theorem 5 . 


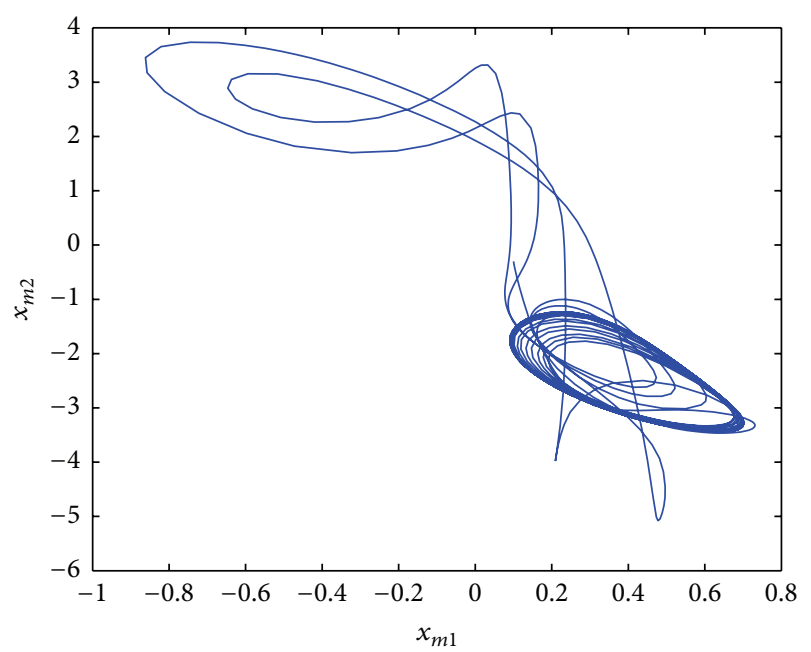

(a) $x_{m}$

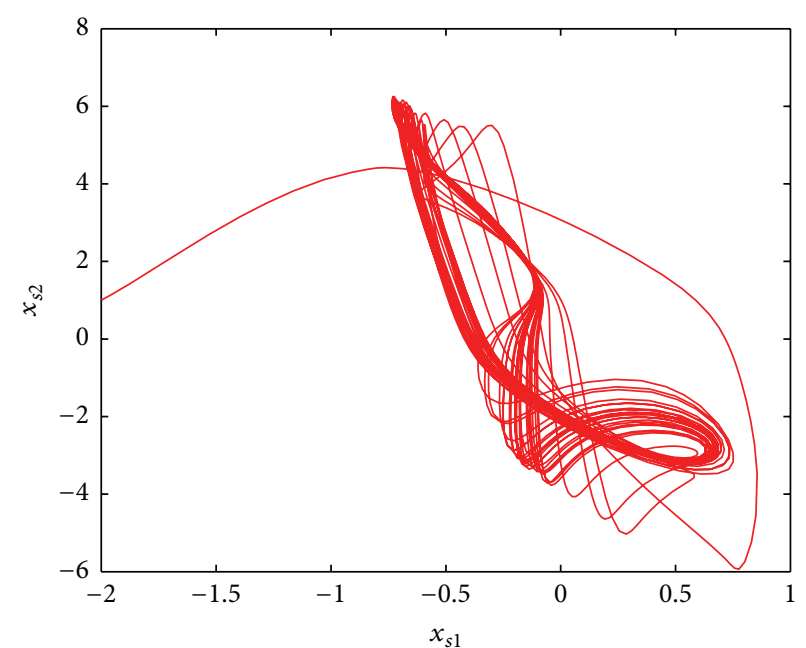

(b) $x_{s}$

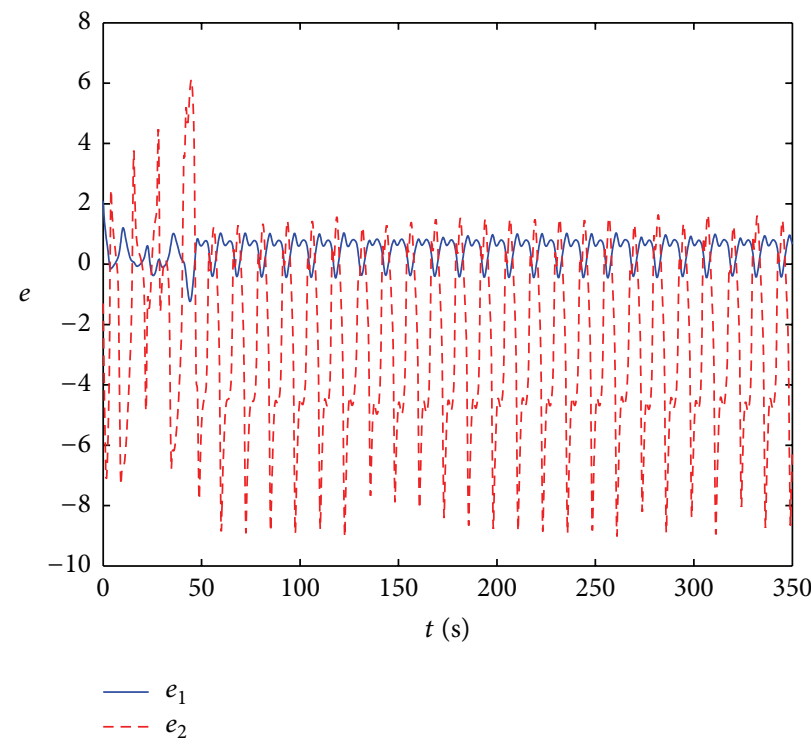

(c) $e(t)$

FIGURE 1: Behavior of the drive and response systems without any control: (a) phase trajectory of drive system, (b) phase trajectory of response system, and (c) synchronization errors.

\section{Simulation Results}

Consider the drive and response system (1) and (2) with parameters:

$$
\begin{aligned}
& A=\left[\begin{array}{cc}
-1 & 0 \\
0 & -1
\end{array}\right], \\
& A_{c}=\left[\begin{array}{ll}
0 & 0 \\
0 & 0
\end{array}\right], \\
& B=\left[\begin{array}{cc}
2 & -0.1 \\
-5 & 4.5
\end{array}\right], \\
& B_{c}=\left[\begin{array}{ll}
-1.5 & -0.1 \\
-0.2 & -4
\end{array}\right],
\end{aligned}
$$

$$
\begin{aligned}
& B_{d}=\left[\begin{array}{cc}
0.001 & 0 \\
0 & 0.001
\end{array}\right], \\
& C=\left[\begin{array}{cc}
1.5 & 0 \\
0 & 0.5
\end{array}\right], \\
& E=\left[\begin{array}{ll}
2 & 0 \\
0 & 2
\end{array}\right], \\
& D=\left[\begin{array}{ll}
1 & 0 \\
0 & 1
\end{array}\right] . \\
& f(t, x(t))=\tanh (x(t)), \\
& g\left(t, x\left(t-d_{1}(t)\right)\right)=\tanh \left(x\left(t-d_{1}(t)\right)\right), \\
& h\left(t, x\left(t-d_{2}(t)\right)\right)=x\left(t-d_{2}(t)\right) \sin (t),
\end{aligned}
$$




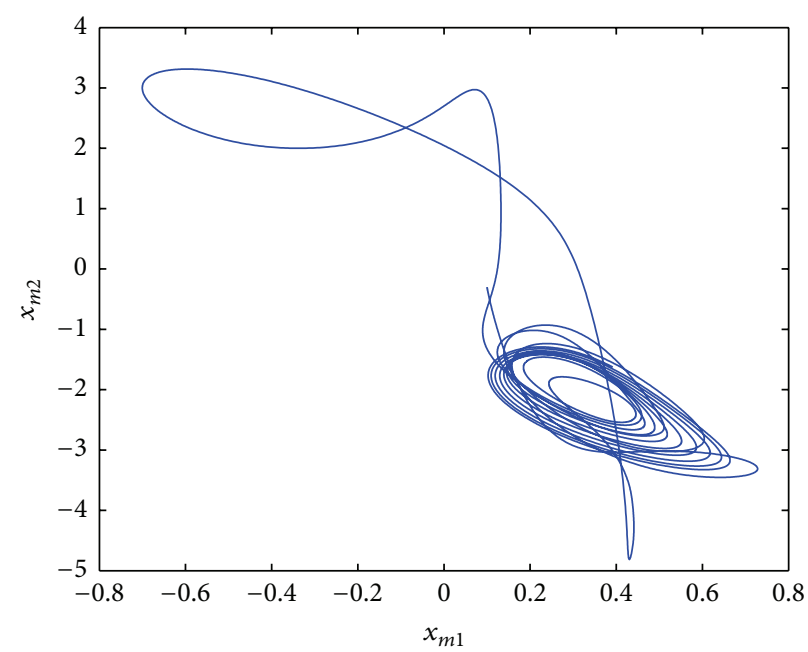

(a) $x_{m}$

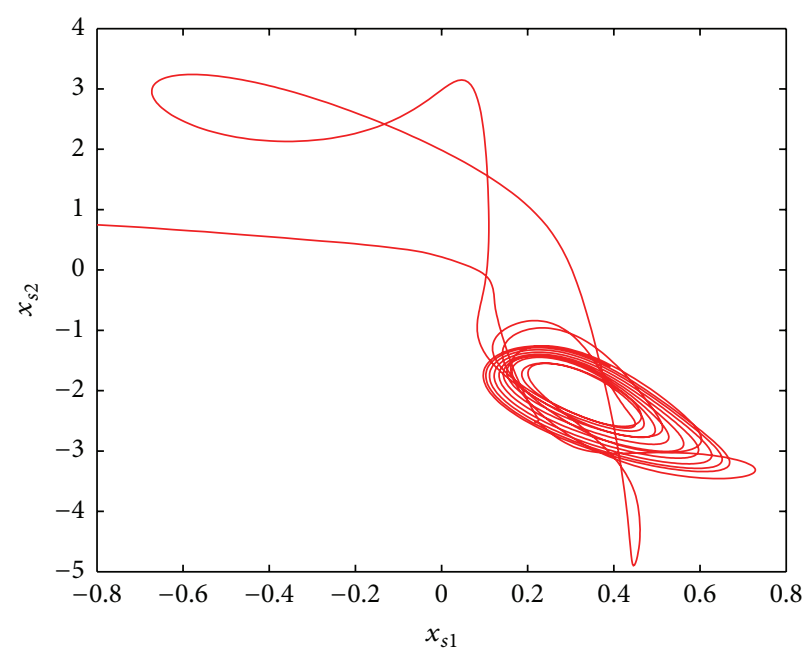

(b) $x_{s}$

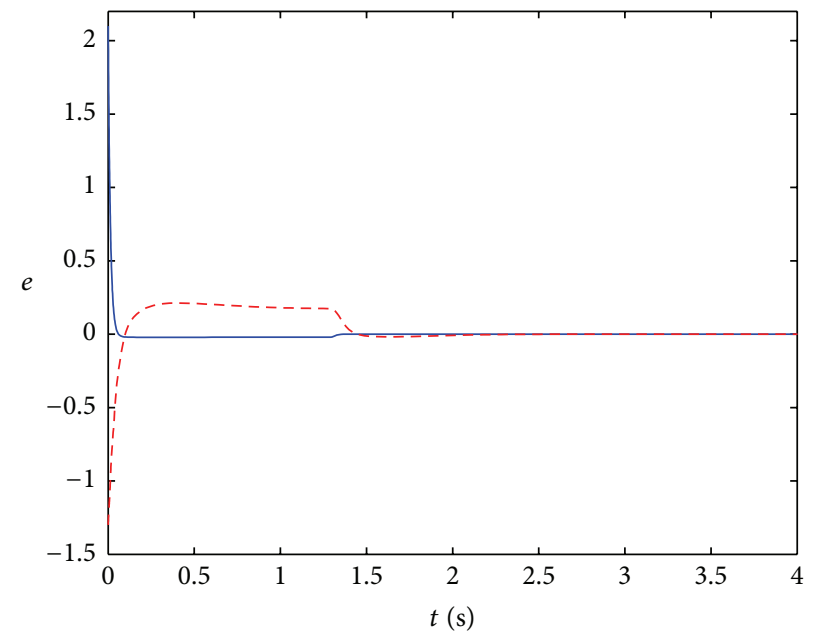

$$
-e_{1}
$$

(c) $e(t)$

FIGURE 2: Behavior of the drive and response systems with controller: (a) phase trajectory of drive system, (b) phase trajectory of response system, and (c) synchronization errors.

$$
\begin{aligned}
& d_{1}(t)=1+0.3 \sin (t), \\
& d_{2}(t)=1-0.02 \sin (10 t), \\
& {\left[\begin{array}{l}
\lambda\left(u_{1}(t)\right) \\
\lambda\left(u_{2}(t)\right)
\end{array}\right]=\left[\begin{array}{c}
1+0.4 \sin \left(u_{1}(t)\right) u_{1}(t) \\
1.2+0.2 \cos \left(u_{2}(t)\right) u_{2}(t)
\end{array}\right],} \\
& \omega(t)=\left[\begin{array}{ll}
0.3 \sin (100 t) & 0.5 \sin (110 t)
\end{array}\right]^{T} .
\end{aligned}
$$

Initial conditions are chosen as

$$
\begin{aligned}
\left(x_{m 1}(0), x_{m 2}(0)\right) & =(0.1,-0.3), \\
\left(x_{s 1}(0), x_{s 2}(0)\right) & =(-2,1), \\
\chi^{*} \rho & =5, \\
\gamma & =0.6 .
\end{aligned}
$$

When $\omega(t)=0$, using the LMI given in Theorem 4 (12), we can obtain

$$
\begin{aligned}
W_{1} & =\left[\begin{array}{cc}
29.1148 & 0.2902 \\
0.2902 & 3.7117
\end{array}\right], \\
W_{2} & =\left[\begin{array}{ll}
27.6971 & 0.2399 \\
0.2399 & 2.9992
\end{array}\right], \\
P & =\left[\begin{array}{cc}
0.7500 & 0 \\
0 & 0.2500
\end{array}\right], \\
\alpha & =46.6242 .
\end{aligned}
$$




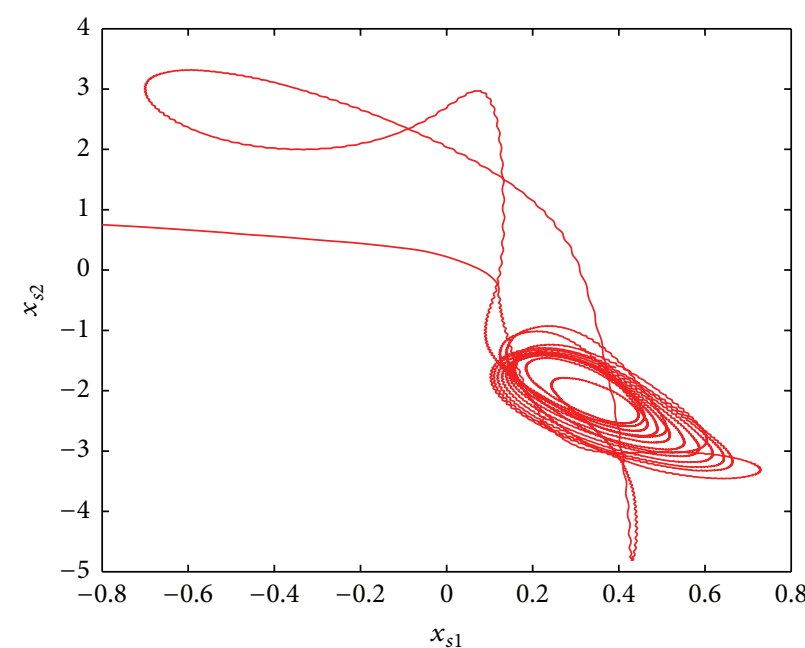

(a) $x_{s}$

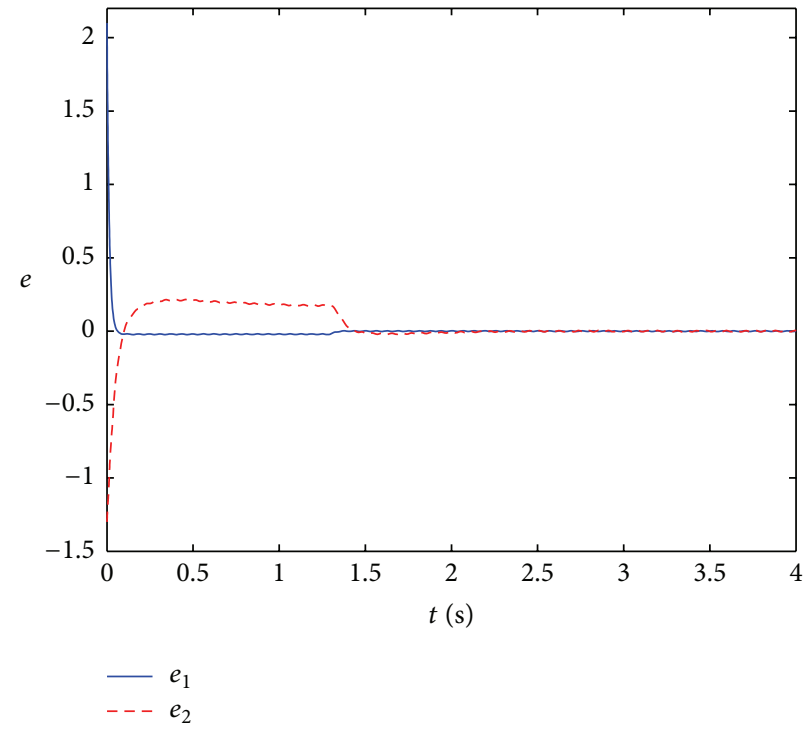

(b) $e(t)$

FiguRE 3: Behavior of the drive and response systems with controller and disturbance: (a) phase trajectory of response system and (b) synchronization errors.

When $\omega(t) \neq 0$, using the LMI given in Theorem 5 (24), we can obtain

$$
\begin{aligned}
W_{1} & =\left[\begin{array}{cc}
48.1311 & 0.2457 \\
0.2457 & 6.9022
\end{array}\right], \\
W_{2} & =\left[\begin{array}{cc}
32.2655 & 0.2254 \\
0.2254 & 6.8700
\end{array}\right], \\
P & =\left[\begin{array}{cc}
0.7500 & 0 \\
0 & 0.2500
\end{array}\right], \\
\alpha & =77.6427 .
\end{aligned}
$$

The phase trajectory of system (1) and (2) without any control is shown in Figures 1(a) and 1(b). The error between them is shown in Figure 1(c). It is obvious in Figure 1 that the systems are nonsynchronous. After application of the proposed controller, the phase trajectory of system (1) and (2) with nonlinear control inputs is shown in Figures 2(a) and 2(b). The error between them is shown in Figure 2(c). It is obvious in Figure 2 that the systems are synchronous. Figure 3(a) illustrates the phase trajectory of response system with nonlinear control inputs and disturbances after applying the proposed controller. Figure 3(b) illustrates that the error signal tends to zero in a short time, in spite of the disturbances.

\section{Conclusion}

We investigate the synchronization problem of chaotic systems with nonlinear control inputs. A robust adaptive controller has been established. By applying this controller, control gain can be regulated automatically and the synchronization of chaotic systems can be achieved. Then, considering external disturbances, we propose a new $H_{\infty}$ synchronization method for chaotic systems. From the above simulation results, we can find that the error signal tends to zero in a short time. Therefore our control strategy is effective in synchronizing chaotic systems.

\section{Conflict of Interests}

The authors declare that there is no conflict of interests regarding the publication of this paper.

\section{Acknowledgment}

The project is supported by the National Natural Science Foundation of China (Grant nos. 61503280, 61403278, and 61272006).

\section{References}

[1] W. Al-Hussaibi, "Effect of filtering on the synchronization and performance of chaos-based secure communication over Rayleigh fading channel," Communications in Nonlinear Science and Numerical Simulation, vol. 26, no. 1-3, pp. 87-97, 2015.

[2] M. Chadli and I. Zelinka, "Chaos synchronization of unknown inputs Takagi-Sugeno fuzzy: application to secure communications," Computers \& Mathematics with Applications, vol. 68, no. 12, pp. 2142-2147, 2014.

[3] J. Q. Yang, Y. T. Chen, and F. Zhu, "Singular reduced-order observer-based synchronization for uncertain chaotic systems subject to channel disturbance and chaos-based secure communication," Applied Mathematics and Computation, vol. 229, pp. 227-238, 2014.

[4] Z. Y. He, K. Li, L. X. Yang, and Y. H. Shi, "A robust digital secure communication scheme based on sporadic coupling chaos 
synchronization," IEEE Transactions on Circuits and Systems I: Fundamental Theory and Applications, vol. 47, no. 3, pp. 397403, 2000.

[5] M. Kalpana, P. Balasubramaniam, and K. Ratnavelu, "Direct delay decomposition approach to synchronization of chaotic fuzzy cellular neural networks with discrete, unbounded distributed delays and Markovian jumping parameters," Applied Mathematics and Computation, vol. 254, pp. 291-304, 2015.

[6] D. Meng, "Neural networks adaptive synchronization for fourdimension energy resource system with unknown dead zones," Neurocomputing, vol. 151, no. 3, pp. 1495-1499, 2015.

[7] J. J. Ohtsubo, "Chaos synchronization and chaotic signal masking in semiconductor lasers with optical feedback," IEEE Journal of Quantum Electronics, vol. 38, no. 9, pp. 1141-1154, 2002.

[8] J. Awrejcewicz, A. V. Krysko, V. Dobriyan, I. V. Papkova, and V. A. Krysko, "Chaotic and synchronized dynamics of non-linear Euler-Bernoulli beams," Computers \& Structures, vol. 155, pp. 85-96, 2015.

[9] A. Göksua, U. E. Kocamazb, and Y. Uyarogluc, "Synchronization and control of chaos in supply chain management," Computers \& Industrial Engineering, vol. 86, pp. 107-115, 2015.

[10] X. J. Lu, H.-X. Li, and M. H. Huang, "Stability and robust design using a sector nonlinearity approach for nonlinear manufacturing systems," Mechanism and Machine Theory, vol. 82, pp. 115-127, 2014.

[11] T. F. Liu, Z.-P. Jiang, and D. J. Hill, "A sector bound approach to feedback control of nonlinear systems with state quantization," Automatica, vol. 48, no. 1, pp. 145-152, 2012.

[12] Y. D. Pan, K. D. Kumar, G. J. Liu, and K. Furuta, "Design of variable structure control system with nonlinear time-varying sliding sector," IEEE Transactions on Automatic Control, vol. 54, no. 8, pp. 1981-1986, 2009.

[13] N. A. Saeed, W. A. El-Ganini, and M. Eissa, "Nonlinear time delay saturation-based controller for suppression of nonlinear beam vibrations," Applied Mathematical Modelling, vol. 37, no. 20-21, pp. 8846-8864, 2013.

[14] M. Eissa, A. Kandil, W. A. El-Ganaini, and M. Kamel, "Vibration suppression of a nonlinear magnetic levitation system via time delayed nonlinear saturation controller," International Journal of Non-Linear Mechanics, vol. 72, pp. 23-41, 2015.

[15] Y.-L. Huang and C.-K. Sun, "Nonlinear saturation behaviors of high-speed p-i-n photodetectors," Journal of Lightwave Technology, vol. 18, no. 2, pp. 203-212, 2000.

[16] T. P. Zhang and S. S. Ge, "Adaptive dynamic surface control of nonlinear systems with unknown dead zone in pure feedback form," Automatica, vol. 44, no. 7, pp. 1895-1903, 2008.

[17] Z. Q. Zhang, S. Y. Xu, and B. Y. Zhang, "Exact tracking control of nonlinear systems with time delays and dead-zone input," Automatica, vol. 52, pp. 272-276, 2015.

[18] C. X. Hu, B. Yao, and Q. F. Wang, "Performance-oriented adaptive robust control of a class of nonlinear systems preceded by unknown dead zone with comparative experimental results," IEEE/ASME Transactions on Mechatronics, vol.18, no. 1, pp. 178189, 2013.

[19] S. C. Tong and Y. M. Li, "Adaptive fuzzy output feedback tracking backstepping control of strict-feedback nonlinear systems with unknown dead zones," IEEE Transactions on Fuzzy Systems, vol. 20, no. 1, pp. 168-180, 2012.

[20] T. Wang, H. Gao, and J. Qiu, "A combined adaptive neural network and nonlinear model predictive control for multirate networked industrial process control," IEEE Transactions on Neural Networks and Learning Systems, 2015.
[21] H. Y. Li, C. W. Wu, P. Shi, and Y. B. Gao, "Control of nonlinear networked systems with packet dropouts: interval type-2 fuzzy model-based approach," IEEE Transactions on Cybernetics, vol. 45, no. 11, pp. 2378-2389, 2014.

[22] H. Li, C. Wu, L. Wu, H.-K. Lam, and Y. Gao, "Filtering of interval type-2 fuzzy systems with intermittent measurements," IEEE Transactions on Cybernetics, 2015.

[23] H. Y. Li, H. J. Gao, P. Shi, and X. D. Zhao, "Fault-tolerant control of Markovian jump stochastic systems via the augmented sliding mode observer approach," Automatica, vol. 50, no. 7, pp. 1825-1834, 2014.

[24] J. H. Park, D. H. Ji, S. C. Won, and S. M. Lee, " $H_{\infty}$ synchronization of time-delayed chaotic systems," Applied Mathematics and Computation, vol. 204, no. 1, pp. 170-177, 2008.

[25] J. H. Kim and H. B. Park, " $H^{\infty}$ state feedback control for generalized continuous/discrete time-delay system," Automatica, vol. 35, no. 8, pp. 1443-1451, 1999.

[26] S. C. Xu and J. Bao, "Distributed control of plant-wide chemical processes with uncertain time-delays," Chemical Engineering Science, vol. 84, pp. 512-532, 2012.

[27] C. H. Zhang, J. N. He, Y. L. Li, X. Y. Li, and P. Li, "Ignition delay times and chemical kinetics of diethoxymethane $/ \mathrm{O}_{2} / \mathrm{Ar}$ mixtures," Fuel, vol. 154, pp. 346-351, 2015.

[28] M.-W. Hong, C.-L. Lin, and B.-M. Shiu, "Stabilizing network control for pneumatic systems with time-delays," Mechatronics, vol. 19, no. 3, pp. 399-409, 2009.

[29] D. Karimipour, S. Pourdehi, and P. Karimaghaee, "Adaptive unstable periodic orbit stabilization of uncertain time-delayed chaotic systems subjected to input nonlinearity," Systems \& Control Letters, vol. 61, no. 12, pp. 1168-1174, 2012.

[30] M. H. Zaheer, M. Rehan, G. Mustafa, and M. Ashraf, "Delayrange-dependent chaos synchronization approach under varying time-lags and delayed nonlinear coupling," ISA Transactions, vol. 53, no. 6, pp. 1716-1730, 2014. 


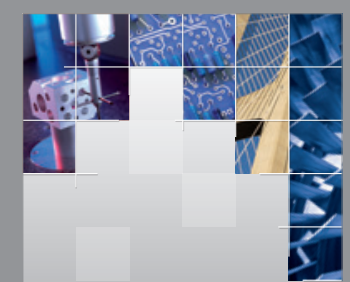

\section{Enfincering}
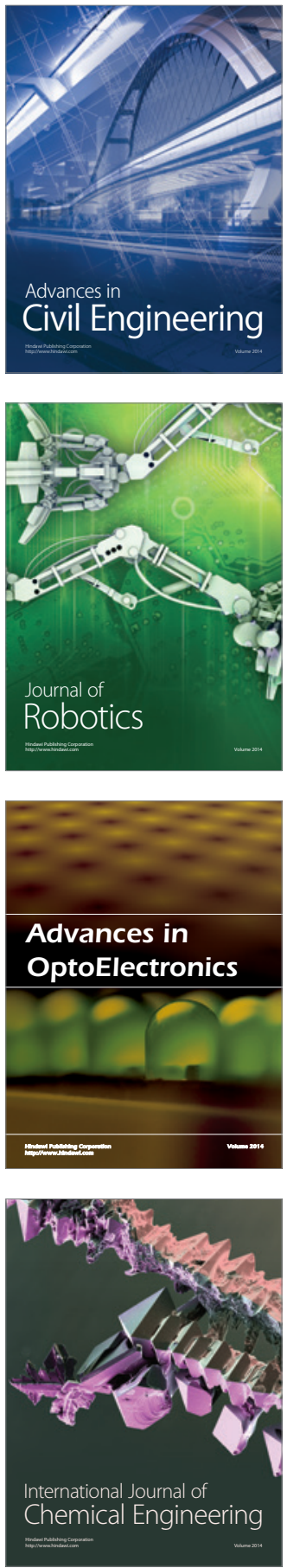

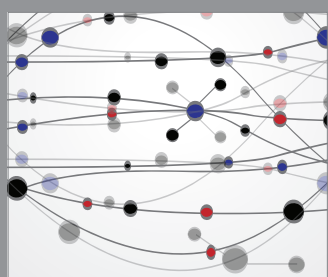

The Scientific World Journal

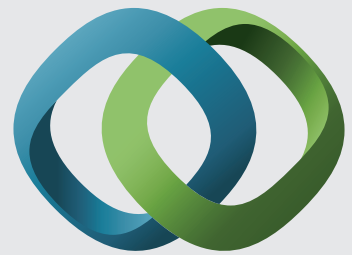

\section{Hindawi}

Submit your manuscripts at

http://www.hindawi.com
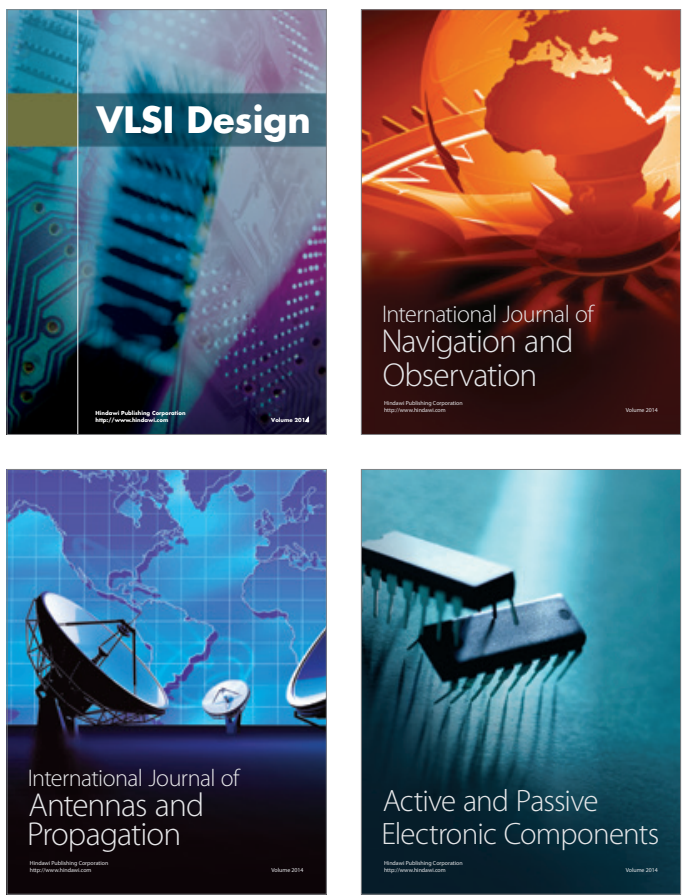
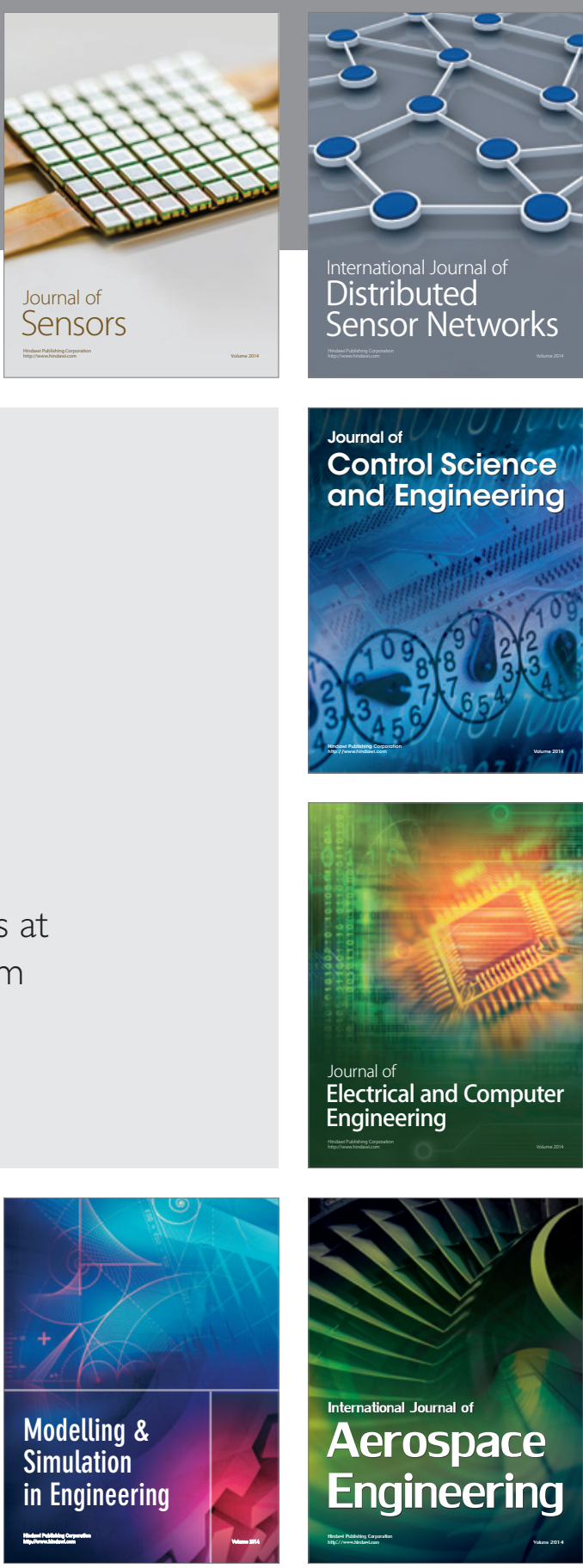

International Journal of

Distributed

Sensor Networks

Journal of

Control Science

and Engineering
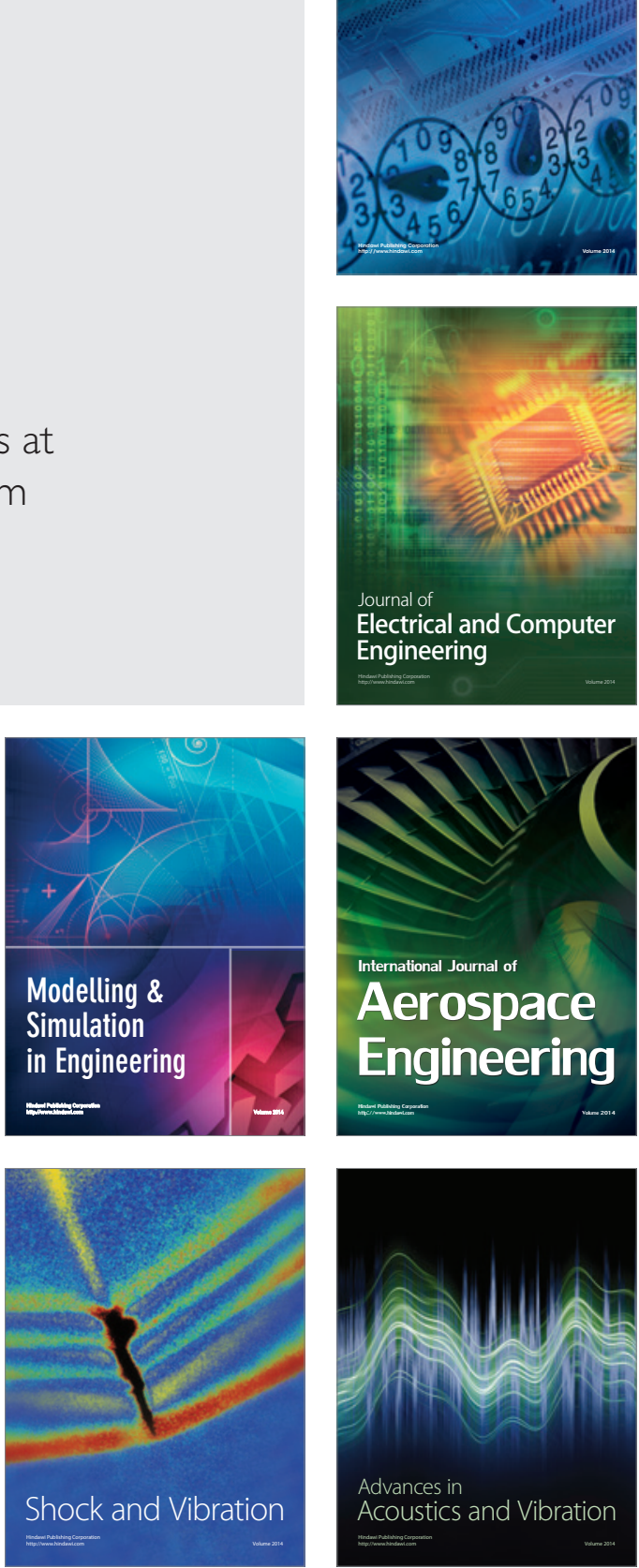